\title{
The effect of aneurysm geometry on the intra-aneurysmal flow condition
}

\author{
Satoshi Tateshima • Aichi Chien • James Sayre • \\ Juan Cebral $\cdot$ Fernando Viñuela
}

Received: 27 December 2009/Accepted: 16 March 2010/Published online: 7 April 2010

(C) The Author(s) 2010. This article is published with open access at Springerlink.com

\begin{abstract}
Introduction Various anatomical parameters affect on intraaneurysmal hemodynamics. Nevertheless, how the shapes of real patient aneurysms affect on their intra-aneurysmal hemodynamics remains unanswered.

Methods Quantitative computational fluid dynamics simulation was conducted using eight patients' angiograms of internal carotid artery-ophthalmic artery aneurysms. The mean size of the intracranial aneurysms was $11.5 \mathrm{~mm}$ (range 5.8 to $19.9 \mathrm{~mm}$ ). Intra-aneurysmal blood flow velocity and wall shear stress (WSS) were collected from three measurement planes in each aneurysm dome. The correlation coefficients $(r)$ were obtained between hemodynamic values (flow velocity and WSS) and the following anatomical parameters: averaged dimension of aneurysm dome, the largest aneurysm dome dimension, aspect ratio, and dome-neck ratio.
\end{abstract}

A part of the results of the present study was presented at the 5th Annual Meeting of the Society of Neuro-interventional Surgery, Olympic Valley, CA, in 2008.

S. Tateshima $(\bowtie) \cdot$ A. Chien $\cdot$ F. Viñuela

Division of Interventional Neuroradiology, David Geffen

School of Medicine at UCLA, University of California,

757 Westwood Plaza Suite 2129A,

Los Angeles, CA 90095-7437, USA

e-mail: stateshima@mednet.ucla.edu

J. Sayre

Department of Biostatistics, School of Public Health,

University of California, Los Angeles,

Los Angeles, CA 90095, USA

J. Cebral

Department of Computational Sciences,

George Mason University,

Fairfax, VA 22030, USA
Results Negative linear correlations were observed between the averaged dimension of aneurysm dome and intraaneurysmal flow velocity $(r=-0.735)$ and also WSS $(r=-0.736)$. The largest dome diameter showed a negative correlation with intra-aneurysmal flow velocity $(r=-0.731)$ and WSS $(r=-0.496)$. The aspect ratio demonstrated a weak negative correlation with the intra-aneurysmal flow velocity $(r=-0.381)$ and WSS $(r=-0.501)$. A clear negative correlation was seen between the intra-aneurysmal flow velocity and the dome-neck ratio $(r=-0.708)$. A weak negative correlation is observed between the intra-aneurysmal WSS and the dome-neck ratio $(r=-0.392)$.

Conclusion The aneurysm dome size showed a negative linear correlation with intra-aneurysmal flow velocity and WSS. Wide-necked aneurysm geometry was associated with faster intra-aneurysmal flow velocity.

Keywords Hemodynamics · Intracranial aneurysm . Computational fluid dynamics simulation .

Aneurysm shape $\cdot$ Aneurysm size

\section{Introduction}

Anatomical parameters of intracranial aneurysms such as aneurysm shape, size, and location clearly correlate with their natural progressions [1-4]. In general, the risk of aneurysm rupture greatly increases as an intracranial aneurysm becomes larger [4]. Past clinical studies also raised a possibility that there was a difference in the aneurysm shape between ruptured and unruptured intracranial aneurysms [1-3]. The International Study on Unruptured Intracranial Aneurysms and many other clinical reports have indicated that the risk of aneurysm rupture varies in anterior and posterior circulations $[4,5]$. 
Intra-aneurysmal hemodynamics is believed to be another important factor related to the etiology and natural history of intracranial aneurysms. Consequently, a variety of intra-aneurysmal hemodynamic studies have been published in the last decade [6-9]. Recent hemodynamic studies of intracranial aneurysms use patient-specific radiographic data, since the geometry of a parent artery and aneurysm itself significantly affect the hemodynamic result. A few studies with the use of patient-specific data have successfully demonstrated statistically significant differences in intra-aneurysmal hemodynamic patterns between ruptured and unruptured intracranial aneurysms $[6,7]$. However, many past hemodynamic studies included intracranial aneurysms with different anatomical parameters (size, shape, and location), which might have obscured the true hemodynamic differences between ruptured and unruptured aneurysms or between stable and unstable aneurysms.

Various anatomical parameters affect on the intraaneurysmal hemodynamics. Nevertheless, a basic question that how the shapes of real patient aneurysms affect on their intra-aneurysmal hemodynamics remains unanswered. Such basic knowledge would greatly help to deeply understand the effect of intra-aneurysmal hemodynamics on the natural history, considering the fact that different size aneurysms carry different rupture risks. In the present study, we examined the hemodynamic characteristics of intracranial aneurysms from a single anatomical location and evaluated the effect of aspect ratio, aneurysm dome size, and domeneck ratio on the intra-aneurysmal hemodynamics.

\section{Materials and methods}

\section{Case selection}

Internal carotid artery-ophthalmic artery (ICA-Oph) aneurysms, which are categorized as side-wall aneurysm, are selected for this hemodynamics simulation study. ICA-Oph aneurysm cases from the three-dimensional (3D) digital subtraction angiography database at the Division of Interventional Neuroradiology, Ronald Reagan UCLA Medical Center were reviewed by interventional neuroradiologists (FV and ST). Only good-quality angiogram (sufficient contrast opacification of all major branches and no patient motions) was considered for the selection of this study. Aneurysms with intraluminal thrombus were excluded from the selection. Ruptured aneurysms with an irregularly shaped sac with contrast stagnation in the sac were also excluded from the selection, since such an aneurysm may have a pseudoaneurysm component in its dome [10]. If a parent artery vasospasm was observed, the case was also excluded from the selection. Moreover, the case with a fetal-type posterior cerebral artery and posterior communicating artery was excluded due to the complexity of outlet flow condition. Thus, eight ICA-Oph aneurysms were selected from the database. There were three ruptured aneurysms and five unruptured aneurysms. Three dimensions of the aneurysm dome (anterior-to-posterior, width, and craniocaudad directions) with the largest size of the aneurysm neck were measured by the interventional neuroradiologists (FV and ST) using the Philips Integris workstation (Philips Medical Systems, Best, The Netherlands). The mean size of the eight ICA-Oph aneurysms was $11.5 \mathrm{~mm}$ (range 5.8 to $19.9 \mathrm{~mm}$ ). The 3D rotational cerebral angiograms were obtained using a Philips Integris unit (Philips Medical Systems, Best, The Netherlands). Data from those images were transferred to the Philips Integris workstation for 3D voxel generation and image restoration.

\section{Computational fluid dynamics simulation}

The 3D voxel data obtained by rotational angiography were then transferred to a Dell 490 hemodynamic analysis workstation in our division. Image-based computational fluid dynamics (CFD) software developed by the Department of Computational Sciences, George Mason University, was utilized for aneurysm flow simulation [6, 7]. The 3D computational model was constructed semiautomatically through segmentation, surface generation, and 3D grid generation for each ICA-Oph aneurysm. The internal carotid artery was reconstructed from the level of the bony carotid canal to the bifurcation of the anterior and middle cerebral arteries. Ophthalmic artery was reconstructed in all cases. Since cases with a large posterior communicating artery were excluded from this study, posterior communicating artery and anterior choroidal artery were not reconstructed.

Normal pulsatile flow conditions acquired from a healthy subject using magnetic resonance phase-contrast measurement (General Electric 1.5 T scanner, Waukesha, WI) were imposed on the CFD simulation [6]. The unsteady incompressible Navier-Stokes equations were implemented and solved under the Newtonian fluid assumption. Blood was assumed to have uniform viscosity of $0.004 \mathrm{~Pa}$ s. Since the accurate information on the pulsatile aneurysm wall motion was not available in 3D rotational angiography, the rigid and no-slip boundary condition was assumed for the aneurysm wall in this CFD study [6-8].

Quantitative hemodynamic analysis

In order to perform a quantitative hemodynamic analysis, intra-aneurysmal blood flow velocity and wall shear stress (WSS) were collected from three measurement planes in each aneurysm: planes crossing the aneurysm dome at the level of the aneurysm neck, middle, and top (Fig. 1). The value of intra-aneurysmal flow velocity and WSS in each 
aneurysm was defined by averaging the flow velocity and WSS data from all of the three measurement planes. The correlation coefficients $(r)$ were obtained by a biostatistician (JS) between the hemodynamic values and the following anatomical parameters: averaged dimension of aneurysm dome, the largest aneurysm dome dimension, aneurysm dome aspect ratio, and dome-neck ratio.

The averaged dimension of the aneurysm dome was defined as an averaged value of anterior-to-posterior, transverse, and craniocaudad dimensions. The aneurysm aspect ratio was defined as aneurysm craniocaudad dimension divided by transverse or anterior-to-posterior dimension, whichever larger. The dome-neck ratio was given as aneurysm width or anterior-to-posterior dimension, whichever larger, divided by the largest aneurysm neck dimension.

\section{Results}

The general characteristics of the hemodynamics patterns in the eight ICA-Oph aneurysms are shown in Fig. 2. This CFD study of patients' aneurysm at a single location shows some correlation between the intra-aneurysmal hemodynamics and such factors as aneurysm diameter, aspect ratio, and dome-neck ratio. Particularly, the averaged values of intra-aneurysmal flow velocity and WSS tend to become smaller as the aneurysm size becomes larger (Fig. 2). In fact, there are negative linear correlations between intraaneurysmal flow velocity and the averaged dimension of aneurysm dome (correlation coefficient: $r=-0.735$ ) and also WSS ( $r=-0.736$; Fig. 3a, b). There is also a negative linear correlation seen between the intra-aneurysmal flow velocity and the largest aneurysm dimension $(r=-0.731$; Fig. $3 c$ ). There is a weak negative correlation between the

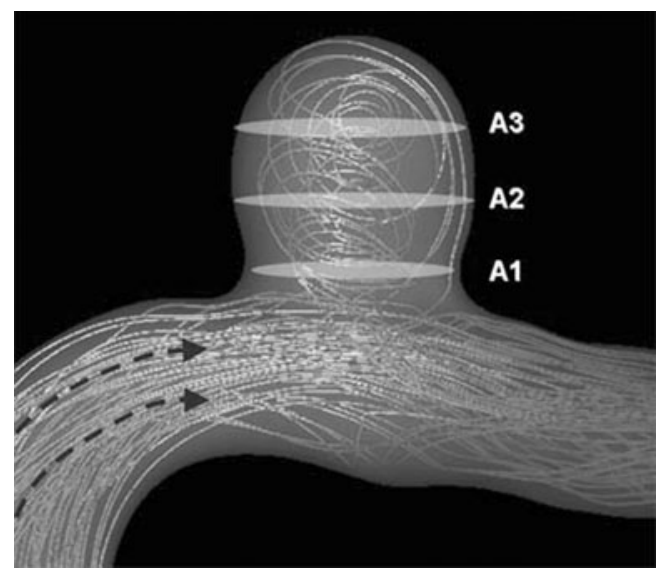

Fig. 1 Schematic representation of planes for the quantitative analyses. Three planes in the aneurysm sac were leveled at the neck $(A 1)$, the middle $(A 2)$, and the top $(A 3)$ of the aneurysm to divide the aneurysm sac into three equal portions intra-aneurysmal WSS and the largest aneurysm dimension with correlation coefficient of -0.496 (Fig. 3d).

The aspect ratio of the aneurysm dome does not show a strong correlation with the intra-aneurysmal flow condition. Some weak negative correlations are observed between the aneurysm aspect ratio and intra-aneurysmal flow velocity $(r=-0.381)$ and WSS $(r=-0.501$; Fig. $3 \mathrm{e}, \mathrm{f})$. There is a clear negative linear correlation between the intra-aneurysmal flow velocity and the dome-neck ratio $(r=-0.708$; Fig. $3 \mathrm{~g})$. In other words, there is a positive linear correlation between the relative size of aneurysm neck and intra-aneurysmal flow velocity. Although wide-necked aneurysms (smaller domeneck ratio) show a faster intra-aneurysmal flow condition, there is only a weak negative correlation observed between the intra-aneurysmal WSS and the dome-neck ratio $(r=-0.392$; Fig. $3 h)$.

\section{Discussions}

We studied the relationship between the shape of intracranial aneurysms and the intra-aneurysmal hemodynamic parameters in a single anatomical location using actual patients' computed tomography angiograms. The result of this study showed that the intra-aneurysmal hemodynamics was significantly affected by such anatomical factors as aneurysm diameter, aspect ratio, and dome-neck ratio. By eliminating other anatomical factors such as aneurysm location, we could demonstrate a clear trend that the intraaneurysmal blood flow was slower, and the averaged value of intra-aneurysmal WSS was lower in the larger aneurysms. In addition, our study showed that there was a weak correlation between the aspect ratio of the aneurysm dome and the intra-aneurysmal hemodynamic values. Some clinical studies found that the aspect ratio of the aneurysm dome was higher in the ruptured aneurysms than that in the unruptured aneurysms [1,3]. Therefore, when a researcher conducts a hemodynamic comparison study in rupture and unruptured aneurysms in order to investigate the hemodynamic risks of aneurysm rupturing, it is critical to match the size of the aneurysms in both groups as well as aspect ratio and dome-neck ratio. Otherwise, such a pre-existing condition, which is the difference in the shape and size of intracranial aneurysms between ruptured and unruptured aneurysm groups, may create a false hemodynamics difference and leads to a false conclusion.

It is not clear whether high shear stress, low shear stress, or high shear stress gradient is associated with the aneurysm wall degeneration [7-9, 11, 12]. Furthermore, it is still controversial whether WSS per se plays a role in the natural history of intracranial aneurysms or not. The result of this study indicates that the larger aneurysms, which is known to carry a higher risk of rupture, showed lower intra- 

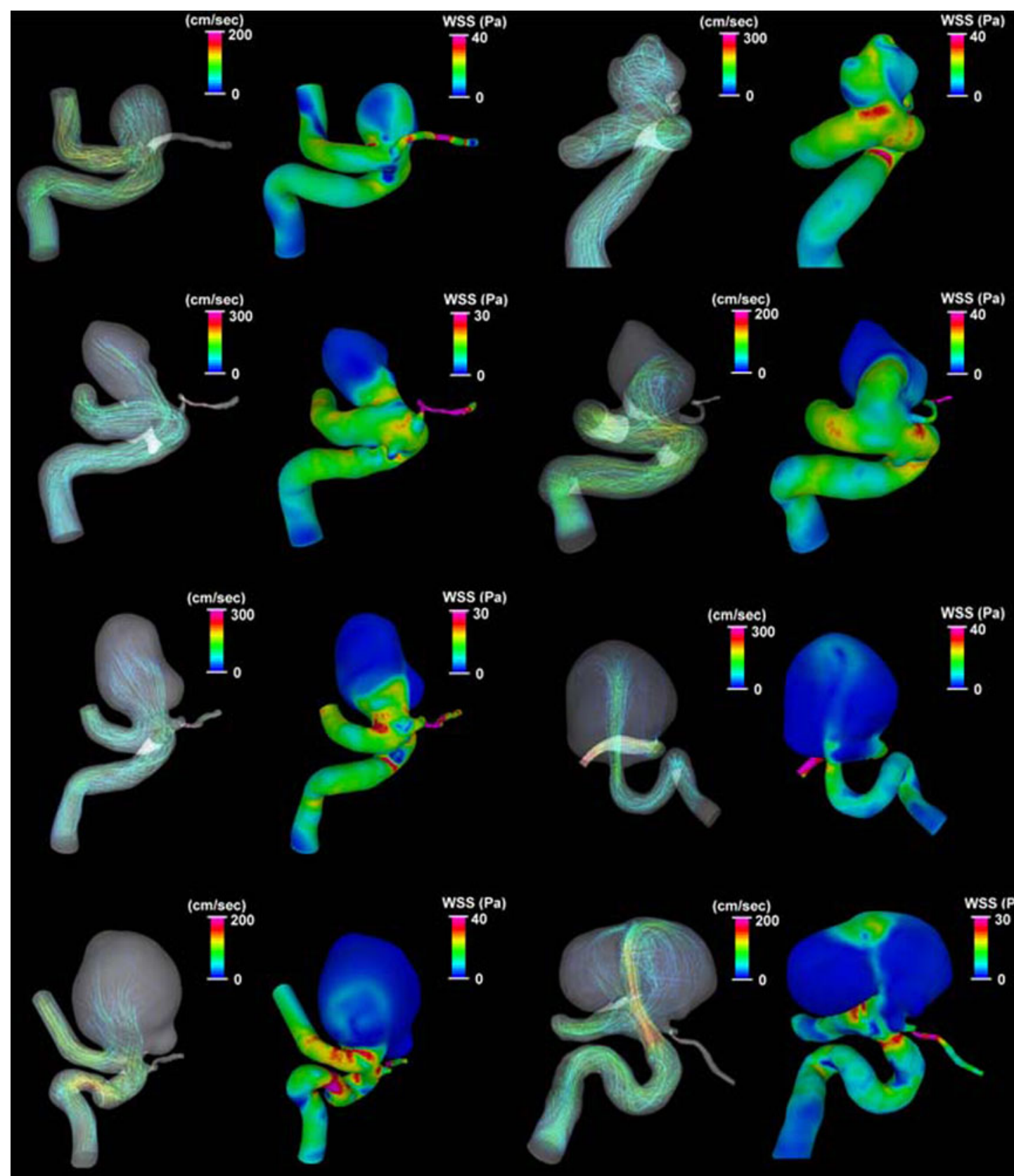

Fig. 2 The general characteristics of the hemodynamics patterns in eight ICA-Oph aneurysms. Blood flow stream line and WSS during peak systolic phase are presented in each ICA-Oph aneurysm. In the

stream line pictures, a red color line indicates faster flow, and a blue one means slower flow. In the WSS distribution pictures, area in red color indicates higher WSS, and area in blue means lower WSS

aneurysmal WSS values. Although it does not directly denote that the low WSS induces the aneurysm rupturing, the low shear condition may be associated with the process of the degeneration of aneurysm walls. A hemodynamic and histopathological comparison study of a large number of ruptured and unruptured aneurysm cases with similar size ranges and shapes in both ruptured and unruptured groups will reveal the true contribution of WSS to the
Fig. $3 \mathbf{a}-\mathbf{h}$ Correlation between the aneurysm shape (dome diameter, aspect ratio, and dome-neck ratio) and flow parameters (velocity and WSS). The number in the right upper corner indicates the correlation coefficients $(r)$. a Correlation between averaged aneurysm dome dimension and velocity, $\mathbf{b}$ between averaged aneurysm dome dimension and WSS, c between the largest dome diameter and velocity, $\mathbf{d}$ between the largest dome diameter and WSS, e between aspect ratio and velocity, $\mathbf{f}$ between aspect ratio and WSS, $\mathbf{g}$ between dome-neck ratio and velocity, and $\mathbf{h}$ between dome-neck ratio and WSS 

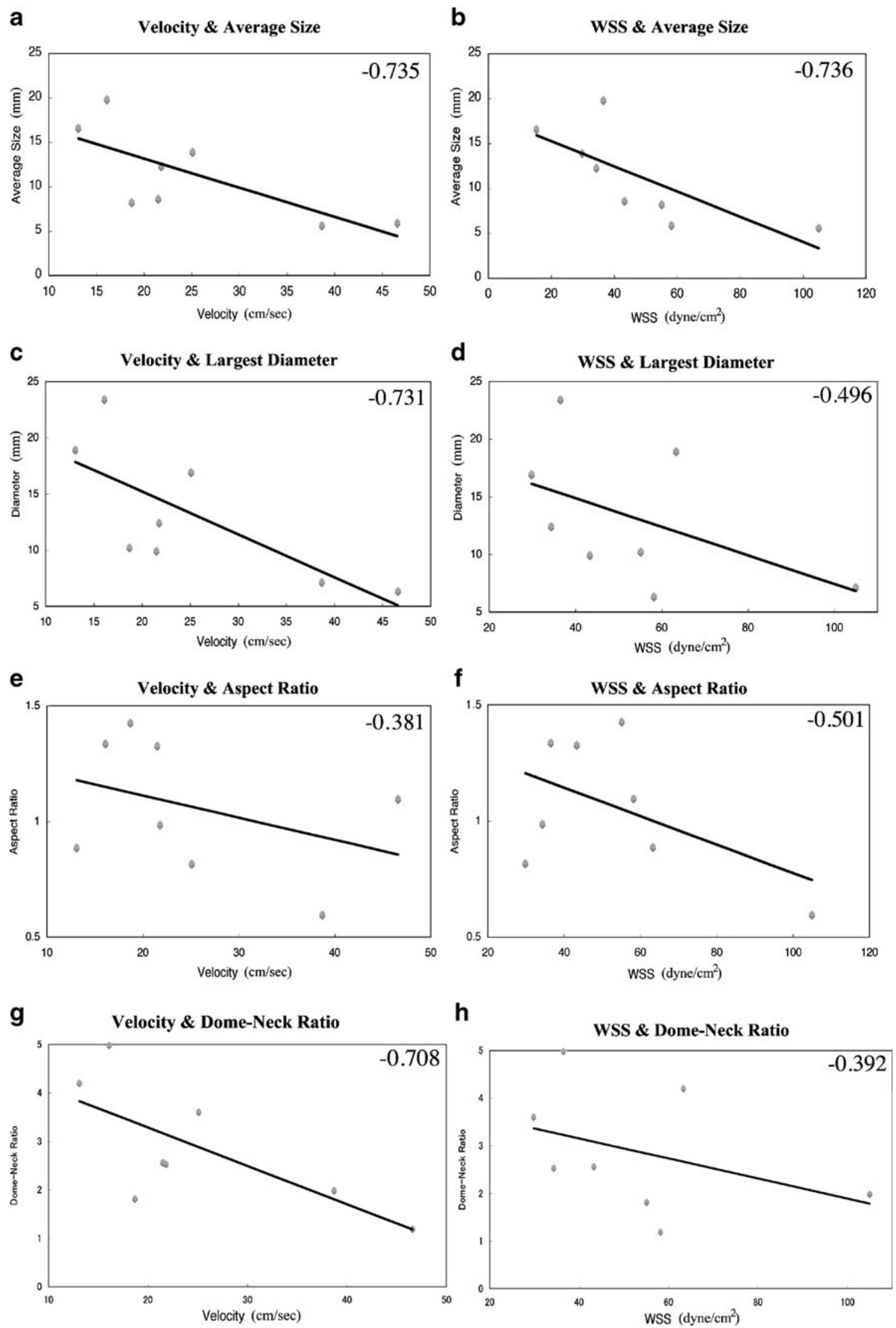
natural progression of intracranial aneurysm. An immunocytochemical/histopathological study of intracranial aneurysms demonstrates that larger aneurysms develop more advanced atherosclerotic lesions on the aneurysm walls than smaller aneurysms [13]. Given that a lower value of WSS is a well-known predisposing factor of atherosclerosis plaque in such arteries as carotid bulb, coronary artery, and aorta, lower WSS in larger intracranial aneurysms may explain the reason why lager aneurysms tend to have more advanced atherosclerotic lesions on them [13-15].

Our study showed an interesting correlation that the intra-aneurysmal blood flow was faster as the aneurysm neck was wider. A larger blood flow inlet relative to the volume of the aneurysm dome in a wide-necked aneurysm may be associated with this finding. Although a major factor for the higher recanalization rate after coil embolization of small/wide-necked aneurysm is an insufficient packing of embolic materials, faster intra-aneurysmal blood flow may also contribute to it [16]. The long-term durability of coil embolization in wide-necked aneurysms seems to be better with the use of self-expandable intracranial aneurysm stents such as Neuroform and Enterprise [16-19]. Again, the primary reason for that must be due to an enhanced packing density with stent assists. However, flow velocity reduction after the placement of aneurysm stents in wide-necked aneurysms may also contribute to the better long-term results [20].

The relationship between aneurysm dome aspect ratio and intra-aneurysmal hemodynamics has been studied in the past [21]. The current study failed to reveal a strong correlation between aspect ratio and such hemodynamic parameters as averaged intra-aneurysmal flow velocity and WSS. Since a higher aspect ratio is known to be associated with a secondary circulation zone in the deeper part of the aneurysm dome, it may correlate with blood flow structure rather than quantitative intra-aneurysmal hemodynamic values [21].

One of the limitations of this study is the fact that it is conducted in a single location. Since aneurysms at a different location may have different hemodynamic characteristics, similar hemodynamic studies in different anatomical locations are mandatory. Creation of a research consortium among centers of excellence in intracranial aneurysm is necessary for a larger hemodynamics study with a large number of aneurysms in each intracranial aneurysm location.

\section{Conclusions}

A quantitative CFD analysis was conducted in eight ICAOph aneurysms with various geometries. The intraaneurysmal hemodynamics was significantly affected by such factors as aneurysm diameter, aspect ratio, and domeneck ratio. The size of the aneurysm dome showed a negative linear correlation with intra-aneurysmal flow velocity and WSS. Wide-necked aneurysm geometry was associated with faster intra-aneurysmal flow velocity.

\section{Conflict of interest None}

Open Access This article is distributed under the terms of the Creative Commons Attribution Noncommercial License which permits any noncommercial use, distribution, and reproduction in any medium, provided the original author(s) and source are credited.

\section{References}

1. Nader-Sepahi A, Casimiro M, Sen J, Kitchen ND (2004) Is aspect ratio a reliable predictor of intracranial aneurysm rupture? Neurosurgery 54:1343-1348

2. San Millán Ruíz D, Yilmaz H, Dehdashti AR, Alimenti A, de Tribolet N, Rüfenacht DA (2006) The perianeurysmal environment: influence on saccular aneurysm shape and rupture. AJNR Am J Neuroradiol 27:504-512

3. Ujiie H, Tamano Y, Sasaki K, Hori T (2001) Is the aspect ratio a reliable index for predicting the rupture of a saccular aneurysm? Neurosurgery 48:495-503

4. Wiebers DO, Whisnant JP, Huston J 3rd, Meissner I, Brown RD Jr, Piepgras DG, Forbes GS, Thielen K, Nichols D, O'Fallon WM, Peacock J, Jaeger L, Kassell NF, Kongable-Beckman GL, Torner JC (2003) International study of unruptured intracranial aneurysms investigators unruptured intracranial aneurysms: natural history, clinical outcome, and risks of surgical and endovascular treatment. Lancet 362:103-110

5. Aarhus M, Helland CA, Wester K (2009) Differences in anatomical distribution, gender, and sidedness between ruptured and unruptured intracranial aneurysms in a defined patient population. Acta Neurochir (Wien) 151(12):1569-1574

6. Cebral JR, Castro MA, Burgess JE, Pergolizzi RS, Sheridan MJ, Putman CM (2005) Characterization of cerebral aneurysms for assessing risk of rupture by using patient-specific computational hemodynamics models. AJNR Am J Neuroradiol 26:2550-2559

7. Chien A, Tateshima S, Castro M, Sayre J, Cebral J, Viñuela F (2008) Patient-specific flow analysis of brain aneurysms at a single location: comparison of hemodynamic characteristics in small aneurysms. Med Biol Eng Comput 46:1113-1120

8. Shojima M, Oshima M, Takagi K, Torii R, Hayakawa M, Katada K, Morita A, Kirino T (2004) Magnitude and role of wall shear stress on cerebral aneurysm: computational fluid dynamic study of 20 middle cerebral artery aneurysms. Stroke 35:2500-2505

9. Tateshima S, Murayama Y, Villablanca JP, Morino T, Nomura K, Tanishita K, Vinuela F (2003) In vitro measurement of fluidinduced wall shear stress in unruptured cerebral aneurysms harboring blebs. Stroke 34:187-192

10. Nomura M, Kida S, Uchiyama N, Yamashima T, Yoshikawa J, Yamashita J, Matsui O (2000) Ruptured irregularly shaped aneurysms: pseudoaneurysm formation in a thrombus located at the rupture site. J Neurosurg 93:998-1002

11. Boussel L, Rayz V, McCulloch C, Martin A, Acevedo-Bolton G, Lawton M, Higashida R, Smith WS, Young WL, Saloner D (2008) Aneurysm growth occurs at region of low wall shear stress: patient-specific correlation of hemodynamics and growth in a longitudinal study. Stroke 39:2997-3002

12. Tateshima S, Tanishita K, Omura H, Villablanca JP, Vinuela $\mathrm{F}$ (2007) Intra-aneurysmal hemodynamics during the growth of an 
unruptured aneurysm: in vitro study using longitudinal CT angiogram database. AJNR Am J Neuroradiol 28:622-627

13. Kosierkiewicz TA, Factor SM, Dickson DW (1994) Immunocytochemical studies of atherosclerotic lesions of cerebral berry aneurysms. J Neuropathol Exp Neurol 53:399-406

14. Ku DN, Giddens DP, Zarins CK, Glagov S (1985) Pulsatile flow and atherosclerosis in the human carotid bifurcation. Positive correlation between plaque location and low oscillating shear stress. Arteriosclerosis 5:293-302

15. Zarins CK, Giddens DP, Bharadvaj BK, Sottiurai VS, Mabon RF, Glagov S (1983) Carotid bifurcation atherosclerosis. Quantitative correlation of plaque localization with flow velocity profiles and wall shear stress. Circ Res 53:502-514

16. Ishii A, Murayama Y, Nien YL, Yuki I, Adapon PH, Kim R, Jahan R, Duckwiler G, Viñuela F (2008) Immediate and midterm outcomes of patients with cerebral aneurysms treated with Matrix 1 and Matrix 2 coils: a comparative analysis based on a single-center experience in 250 consecutive cases. Neurosurgery 63:10711079
17. Lubicz B, Bandeira A, Bruneau M, Dewindt A, Balériaux D, De Witte O (2009) Stenting is improving and stabilizing anatomical results of coiled intracranial aneurysms. Neuroradiology 51:419-425

18. Sedat J, Chau Y, Mondot L, Vargas J, Szapiro J, Lonjon M (2009) Endovascular occlusion of intracranial wide-necked aneurysms with stenting (Neuroform) and coiling: mid-term and long-term results. Neuroradiology 51:401-409

19. Piotin M, Blanc P, Spelle L, Mounayer C, Piantino R, Schmidt PJ, Moret J (2010) Stent-assisted coiling of intracranial aneurysms. Clinical and angiographic results in 216 consecutive aneurysms. Stroke 41:110-115

20. Tateshima S, Tanishita K, Hakata Y, Tanoue SY, Viñuela F (2009) Alteration of intraaneurysmal hemodynamics by placement of a self-expandable stent. J Neurosurg 111:22-27

21. Ujiie $\mathrm{H}$, Tachibana $\mathrm{H}$, Hiramatsu $\mathrm{O}$, Hazel AL, Matsumoto $\mathrm{T}$, Ogasawara Y, Nakajima H, Hori T, Takakura K, Kajiya F (1999) Effects of size and shape (aspect ratio) on the hemodynamics of saccular aneurysms: a possible index for surgical treatment of intracranial aneurysms. Neurosurgery 45:119-130 